\title{
Morphological variation of the velum in children and adults using magnetic resonance imaging
}

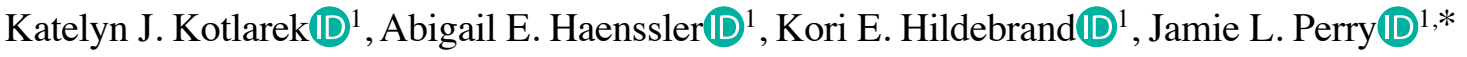 \\ ${ }^{1}$ Department of Communication Sciences and Disorders, East Carolina University, Greenville, NC, USA
}

\section{ABSTRACT}

\begin{abstract}
Purpose: The purpose of this study was to investigate variations in velar shape according to age, sex, and race using magnetic resonance imaging (MRI).

Materials and Methods: The study sample consisted of 170 participants ( 85 children, 85 adults) between 4 and 34 years of age. Velar morphology was visually classified using midsagittal MRI scans for each participant by 2 independent raters. Inter- and intra-rater reliability was assessed. Statistical analyses were performed to identify correlations of velar shape with sex, age, and race.

Results: The most frequent velar shape was "butt" for both adults (41\%) and children (58\%) in this study. The least common shapes for adults were "leaf" and "S." The children did not exhibit any "leaf" or "straight" velar shapes. A statistically significant difference was noted for age with respect to velar shape $(P=0.014)$. Sex and race were found to have no significant impact on velar shape in this study.

Conclusion: When using MRI to evaluate velar morphology, the "butt" shape was most common in both children and adults. Velar shape varied significantly with age, while race and sex did not have a significant impact. (Imaging Sci

Dent 2019; 49: 153-8)
\end{abstract}

KEY WORDS: Magnetic Resonance Imaging; Palate, Soft; Pharynx

\section{Introduction}

The velum, or soft palate, is a muscular structure located immediately posterior to the hard palate and contained within the pharynx. The posterior and lateral pharyngeal walls, in conjunction with the velum, form a muscular valve called the velopharyngeal port. The primary function of this muscular valve is to close off the oral cavity from the nasal cavity during oral speech tasks and swallowing. Anatomical deficiencies, such as cleft palate, are known to cause velopharyngeal insufficiency, or the inability of the velopharyngeal mechanism to completely close off the oral cavity from the nasal cavity. The literature has shown that

*This study was made possible by grant numbers 1R03DC009676-01A1 from the National Institute on Deafness and Other Communication Disorders and 1F31DE 027878-01 from the National Institute of Dental and Craniofacial Research. Its contents are solely the responsibility of the authors and do not necessarily represent the official views of the National Institutes of Health.

Received February 1, 2019; Revised March 14, 2019; Accepted April 10, 2019

* Correspondence to: Prof. Jamie L. Perry

Department of Communication Sciences and Disorders, 3310V College of Allied

Health Sciences, Mail Stop 668, East Carolina University, Greenville, NC 27834, USA

Tel) 1-252-744-6144,E-mail) perryja@ecu.edu velar shape may be pertinent to patient health and well-being, specifically relative to obstructive sleep apnea (OSA) ${ }^{1}$ and oral submucous fibrosis. ${ }^{2-5}$

You et al. ${ }^{6}$ established a qualitative classification system to describe the shape of the velum across 200 participants ranging from 5 to 48 years old using cephalometric images. ${ }^{6}$ Participants were classified as having the following 6 distinct velar shapes: "straight," "rat-tail," "crook," "S," "butt," and "leaf." The "rat-tail" and "butt" shapes were more common in men than women, and the "crook" shape was only observed in the pediatric $(<18$ years of age) population. In contrast, adults were more likely to have a "leaf"-shaped velum. Growth of the velum as children mature is believed to be the reason why certain shapes occur more often in adults. ${ }^{6}$ However, because the age group of young children included participants from 5 to 18 years of age, it is difficult to draw conclusions regarding age effects within the sample analyzed by You et al. ${ }^{6}$

Additional studies have applied qualitative velar classifications to analyze lateral cephalograms. ${ }^{1-4,7-14}$ For the 
majority of these studies, the most common velar shape was "leaf," and no significant difference in shape was reported based on sex or age. ${ }^{1,7,9,12,15}$ Praveen et al. ${ }^{11}$ also found no significant difference in velar shape according to sex, but determined that the "rat-tail" shape was most common among both males and females. However, Kumar and $\mathrm{Gopal}^{8}$ and Verma et al. ${ }^{14}$ found the most common shape to be "leaf," with a strong correlation with sex and/or age groups. Across the literature, variability exists regarding the impact of sex and age on velar morphology.

Agrawal et al. ${ }^{16}$ and More et al. ${ }^{17}$ utilized the classification system from You et al. ${ }^{6}$ to analyze velar morphology using computed tomography (CT) images. This imaging technique has the advantage of providing a true midsagittal image without the potential for interference from adjacent anatomy (e.g., the mandible), as would be seen with lateral cephalometry. Agrawal et al. ${ }^{16}$ found that the morphological differences between the velar shape of male and female participants were not significant, but velar shape varied significantly across age groups, which ranged from 15 to 45 years of age in 5-year increments. The most common shape found in that study was "rat-tail."16 On the contrary, More et al. ${ }^{17}$ found the morphology of the velum to show a nonsignificant association with age, and the most common shape was "butt." Both studies ${ }^{16,17}$ found no significant relationship with sex.

The prevalence of specific velar shapes has been shown to vary across regional groups within India and China. In the central Madhya Pradesh population of India, the most common shape was "rat-tail." 16 Within a north Indian sub-population, the most common velar shape was "leaf." ${ }^{, 6,14}$ Niu et al. ${ }^{10}$ completed a similar investigation within a normative Chinese population and also found "leaf" to be the most common shape. Research has shown that craniofacial and velopharyngeal dimensions vary based on race across African-American, Asian, and Caucasian individuals. ${ }^{18}$

To the best of our knowledge, race-, age-, and sex-based effects on velar shape have not been controlled within the same study, which may contribute to a greater reported occurrence of one velar shape over another. In addition, cephalometric imaging produces a sagittal image that can be masked by adjacent and overlapping anatomy (e.g., the mandible) and is sensitive to head rotation, which can make assessments of velar shape less reliable than when CT or magnetic resonance imaging (MRI) are used. MRI offers the advantage of a single, midsagittal image plane with higher image resolution than radiographic imaging methods. Selecting the midsagittal image allows the examiner to modify the slice selection to remove the effect of head rotation on the data. Although radiographic methods have previously been utilized due to cost-effectiveness and ease of use, ${ }^{12}$ MRI provides better contrast of muscular and soft tissue structures than radiological imaging assessments, which may reduce the high level of variability observed in the literature on velar morphology. ${ }^{19}$ To the best of our knowledge, this is the first study to use MRI to describe the shape of the velum. The purpose of this study was to investigate the morphological variation of the velum using MRI across different age, sex, and racial groups.

\section{Materials and Methods}

In accordance with the local Institutional Review Boards, 170 participants were recruited for participation in this study. Participants were excluded if they had a history of previous surgery or a medical condition affecting their head and neck anatomy. There were 85 adult participants between 18 and 34 years of age and 85 child participants between 4 and 18 years of age. The participants varied in terms of both sex ( 83 male, 87 female) and race ( 80 Caucasian, 52 African-American, 32 Asian, 2 Hispanic, and 3 interracial). Using a Siemens 3 Tesla Trio (Erlangen, Germany) and a 12-channel Siemens Trio head coil, a high-resolution T2-weighted turbo-spin-echo 3-dimensional (3D) anatomical scan with high sampling efficiency was completed with the participants lying in the supine position without sedation. To ensure that the velum was in a relaxed and lowered position, participants were instructed to breathe through their nose with a closed mouth. A large

Table 1. Velar shape configurations and definitions, as described by You et al. ${ }^{6}$

\begin{tabular}{ll}
\hline Shape & \multicolumn{1}{c}{ Definition } \\
\hline Leaf & the medial portion of the velum is thicker than the posterior potion of the velum \\
Rat-tail & the size of the velum is consistent from the anterior to the posterior portion, gradually narrowing to a point \\
$\mathrm{S}$ & the velum is distorted from the hard palate to the uvula and contains two bends \\
Butt & the length of the velum is consistent with a blunt end \\
Crook & the velum appears to have a crook that hooks anteriorly and superiorly \\
Straight & the velum is thin in size with a straight-line shape \\
\hline
\end{tabular}


field of view $(25.6 \times 19.2 \times 15.5 \mathrm{~cm})$ was covered with an 0.80 isotropic resolution in 4 minutes and 52 seconds. This imaging sequence has been described in the previous literature. $^{20,21}$

The MRI data was transferred into Thermo Scientific ${ }^{\mathrm{TM}}$ Amira $^{\mathrm{TM}}$ Software (Thermo Fisher Scientific, Waltham, MA, USA). This software has a native Digital Imaging and Communication in Medicine support program that preserves the natural geometric configuration of the images. To visualize the velum, the 3D data were sliced along a midsagittal plane. All midsagittal MRI scans were randomized and then rated blindly by 2 raters to establish inter-rater reliability. Intra-rater reliability was assessed by the primary rater performing a re-evaluation at an interval of 3 weeks between ratings. The 6 velar shape configurations described by You et al. ${ }^{6}$ were utilized in this study, as described in Table 1.

Statistical analyses were conducted using SPSS version 25.0 (IBM Corp., Armonk, NY, USA). An analysis of variance (ANOVA) was used to determine the significance of differences in velar shape based on sex, age, and race. Inter-rater and intra-rater reliability measures were obtained using the intra-class correlation coefficient to determine the consistency of measurements between raters and for the primary rater. Estimates were based on a 2-way random model with a $95 \%$ confidence interval.

\section{Results}

The frequency of velum shapes across groups is presented in Table 2. Using an ANOVA, a statistically significant difference was noted for age $(F(1,158)=6.146$,
$P=0.014)$. No significant difference was observed for sex $(\mathrm{F}(1,158)=1.407, P=0.237)$ or $\operatorname{race}(\mathrm{F}(4,158)=0.458$, $P=0.766)$. Adults presented with all 6 shapes, with the majority having with a "butt" (41.2\%) or "crook" (30.6\%) shape (Fig. 1). Children did not present with any cases of a "leaf" or "straight" velar shape (Fig. 2). Instead, children typically presented with a "butt" (57.6\%) or "rat-tail" (28.2\%) velum. The distribution of velar shapes across both adults and children is visually depicted in Figure 3. With respect to sex, a "butt" shape was predominant in both males $(54.2 \%)$ and females $(44.8 \%)$. No males displayed a "leaf" shape, and no females had an "S" shape. In the analysis based on race, a "butt" shape was predominant

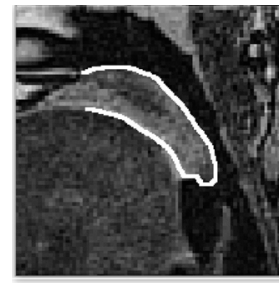

Leaf

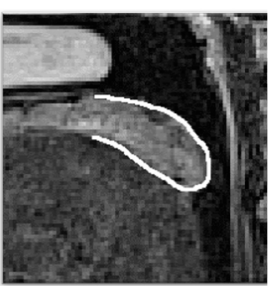

Butt

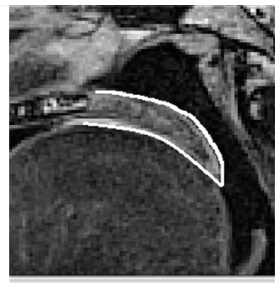

Rat-tail

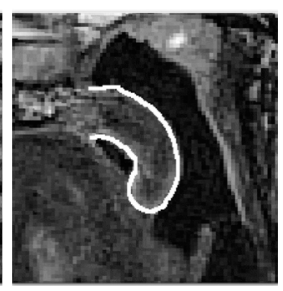

Crook

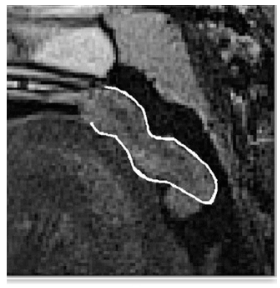

$\mathbf{S}$

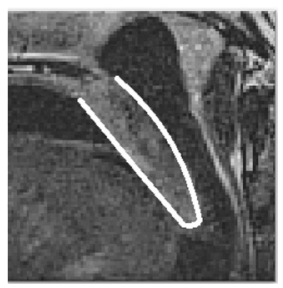

Straight
Fig. 1. Midsagittal magnetic resonance images of adults show all 6 morphological categories of velar shape. The velum is outlined in white to emphasize its shape and to differentiate it from the surrounding structures.

Table 2. Frequency of velum shapes based on groups and results of ANOVA

\begin{tabular}{|c|c|c|c|c|c|c|c|c|c|}
\hline \multirow[t]{2}{*}{ Group } & \multirow[t]{2}{*}{ Subgroups } & \multirow[t]{2}{*}{ Leaf } & \multirow[t]{2}{*}{ Rat-tail } & \multirow[t]{2}{*}{$\mathrm{S}$} & \multirow[t]{2}{*}{ Butt } & \multirow[t]{2}{*}{ Crook } & \multirow[t]{2}{*}{ Straight } & \multicolumn{2}{|c|}{$\begin{array}{l}\text { Test for group } \\
\text { differences }^{\dagger}\end{array}$} \\
\hline & & & & & & & & $\mathrm{F}$ & $P$-value \\
\hline \multirow[t]{2}{*}{ Sex } & $\operatorname{Male}(\mathrm{n}=83)$ & $0 \%$ & $18.1 \%$ & $1.2 \%$ & $54.2 \%$ & $22.9 \%$ & $3.6 \%$ & 1.407 & .237 \\
\hline & Female $(n=87)$ & $1.1 \%$ & $32.2 \%$ & $2.3 \%$ & $44.8 \%$ & $19.5 \%$ & $0 \%$ & & \\
\hline \multirow[t]{2}{*}{ Age } & $\begin{array}{l}\text { Child (4-18 years) } \\
(n=85)\end{array}$ & $0 \%$ & $28.2 \%$ & $2.4 \%$ & $57.6 \%$ & $11.8 \%$ & $0 \%$ & 6.146 & $.014^{*}$ \\
\hline & $\begin{array}{l}\text { Adult }(18-34 \text { years }) \\
(\mathrm{n}=85)\end{array}$ & $1.2 \%$ & $22.4 \%$ & $1.2 \%$ & $41.2 \%$ & $30.6 \%$ & $3.5 \%$ & & \\
\hline \multirow[t]{5}{*}{ Ethnicity } & Caucasian $(\mathrm{n}=80)$ & $0 \%$ & $25.0 \%$ & $0 \%$ & $55 \%$ & $18.8 \%$ & $1.2 \%$ & .458 & .766 \\
\hline & $\begin{array}{l}\text { African American } \\
(\mathrm{n}=52)\end{array}$ & $1.9 \%$ & $19.2 \%$ & $3.8 \%$ & $53.8 \%$ & $17.3 \%$ & $3.8 \%$ & & \\
\hline & $\operatorname{Asian}(n=32)$ & $0 \%$ & $37.5 \%$ & $0 \%$ & $28.1 \%$ & $34.4 \%$ & $0 \%$ & & \\
\hline & Hispanic $(\mathrm{n}=2)$ & $0 \%$ & $0 \%$ & $0 \%$ & $100 \%$ & $0 \%$ & $0 \%$ & & \\
\hline & Interracial $(\mathrm{n}=3)$ & $0 \%$ & $33.3 \%$ & $33.3 \%$ & $33.3 \%$ & $0 \%$ & $0 \%$ & & \\
\hline
\end{tabular}

$* P<0.05,{ }^{\dagger}$ ANOVA 


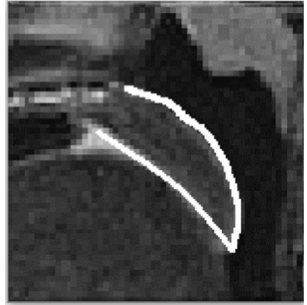

Rat-tail

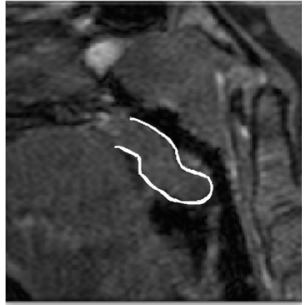

$\mathbf{S}$

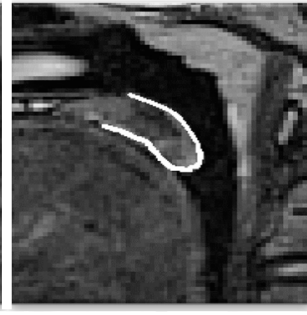

Butt

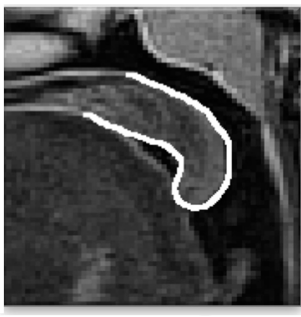

Crook

Fig. 2. Midsagittal magnetic resonance images of children show 4 morphological categories of velar shape. The velum is outlined in white to emphasize its shape and to differentiate it from the surrounding structures. The "leaf" and "straight" shapes are not shown because there were no instances of these types within the sample of children analyzed in this study.

in the Caucasian (55.0\%), African American (53.8\%), Interracial (33.0\%), and Hispanic (100\%) participants. The "rattail" shape was predominantly present in the Asian population $(37.5 \%)$. Inter-rater reliability ranged from 0.639 to 0.785 , indicating good reliability between raters. Intra-rater reliability ranged from 0.673 to 0.807 , indicating good reliability between repeated measures.

\section{Discussion}

You et al. ${ }^{6}$ suggested the likelihood of velar shape being specific to age. The findings of the present study support the hypothesis that velar shape shows significant differences according to age. The children within this study were more likely to have a "butt" or "rat-tail" velar shape, whereas adults were more likely to have a "butt" or "crook" shape. In contrast, You et al. ${ }^{6}$ reported that the "crook" shape only occurred in children, while adults were more likely to have a "leaf"-shaped velum. Changes in velar shape are likely due to growth of the velum as children mature. Previous studies have reported that growth led to increased velar length and velar thickness. ${ }^{22,23}$ These anatomical changes likely result in the presence of certain velar shapes in adults.

Ratings using the categorization of velar shape developed by You et al. ${ }^{6}$ demonstrated good inter- and intra-rater reliability in the present study. The highest inter-rater agreement was for ratings of the "crook" velar shape, which could be attributed to this shape having the most distinctive feature (a hook-like appearance at the end of the velar body). The velar shape configurations with the lowest frequency in this study were the "leaf" and "straight" shapes. This finding was consistent with respect to both inter- and intra-rater reliability.

Although the results of the present study demonstrated good reliability using the classification of You et al., ${ }^{6}$ further analysis is necessary to determine the clinical significance of velar shape and which anatomical variables impact velar shape. Specifically, future research should determine whether velar shape is correlated to the velopharyngeal closure pattern. This is of clinical interest for individuals with cleft palate, as secondary surgical intervention techniques for velopharyngeal dysfunction are typically selected based on the length and movement of the velum relative to the lateral pharyngeal walls. You et al. ${ }^{6}$ proposed that surgical failure of primary palatoplasty may result from variation in velar tissue morphology. A preliminary assessment of 15 participants with cleft palate showed that the "butt" shape was predominant within this population, whereas the "lanceolate" ("leaf") shape was most predominant in the non-cleft population. ${ }^{1}$ The "butt" shape was predominant among the adult and child participants in the present study, none of whom had cleft palate. In the future, the velar shape of individuals with cleft palate should be analyzed, controlling for race, sex, and age and using a larger sample size. The normative values found in this study should be compared to specific clinical populations, such as individuals with OSA or cleft palate.

Pepin et al. ${ }^{5}$ reported that a "hooked" or "S-shaped" velum predicted a higher risk of OSA, while Deepa et al. ${ }^{1}$ reported a higher prevalence of a "lanceolate" ("leaf") shaped palate with a longer velum in individuals with OSA. Research has shown that the prevalence of OSA increases with advancing age, male sex, and higher body mass index. ${ }^{24}$ The population prevalence has been estimated to be between $9 \%$ to $38 \% .{ }^{24}$ In contrast, the prevalence of sleep-disordered breathing was found to be $1.5 \%$ in elementary school children aged $5-7 . .^{25}$ In the present study, the combined prevalence of "leaf," "crook," and " $\mathrm{S}$ " shapes was lower in children (14\%) than in adults (33\%), which might partially explain why OSA is more common in adults than in children. The present study did not assess whether 


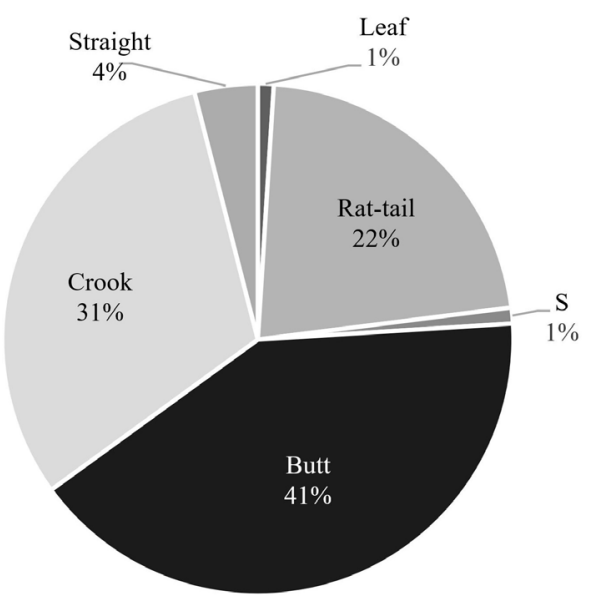

A

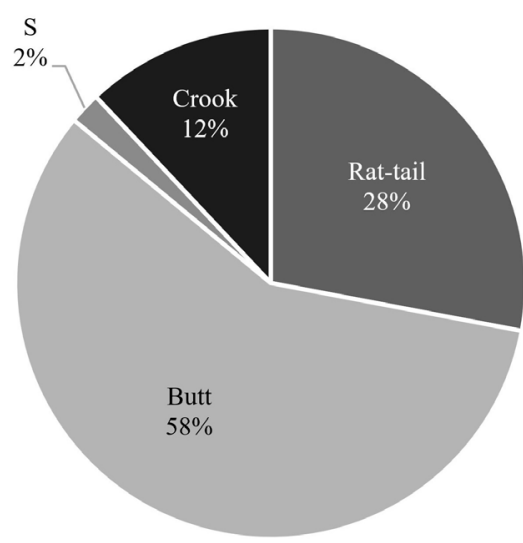

B

Fig. 3. Pie charts depicting the distribution of each velar shape category in adults (A) and children (B).

participants had signs or symptoms of OSA, which is a limitation. Future research should employ a larger sample size to investigate velar morphology among participants diagnosed with OSA.

Age was a significant predictor of velar morphology in this study, in agreement with other reports. ${ }^{14,16}$ A group of participants between 4 and 34 years of age was utilized to capture a period of increased skeletal growth, while encompassing both child and adult participants. Utilization of only 2 groups (children and adults) is a limitation of the present study. The literature on this topic has found age to be a significant predictor of velar shape when participants were divided into age groups spanning 5 years, ${ }^{7,14,16}$ but not when divided into age groups spanning 10 years. ${ }^{15,17}$ Future research should analyze groups of participants at each age to determine which ages are associated with changes in velar morphology. It is reasonable to believe that the greatest morphological change of the velum may take place during puberty. Consideration should be given to following participants longitudinally to determine how velar shape changes over time within the same individual.

To our knowledge, this is the first investigation of velar morphology using MRI. The previous literature on this topic has employed radiographic methods. MRI is typically performed with the participant in the supine position, as was done in this study, while radiographic imaging methods are performed with the participant in the upright position. Previous literature has shown that gravity does not influence the resting velar position or the degree of elevation and retraction of the velum during speech; results have demonstrated that differences due to posture are likely negligible. ${ }^{26,27}$ However, neither of those studies specifically analyzed changes in velar shape. Future research should investigate changes in velar morphology secondary to changes in body positioning to determine whether gravity has an impact on velar shape.

In conclusion, our study was the first to utilize MRI to study velar morphology in both adults and children. In both of these groups, the most frequent velar shape was "butt." The least common shapes in the adult group were "leaf" and "S." None of the children exhibited "leaf" or "straight" velar shapes. Velar morphology varied significantly with age, while race and sex did not have a significant impact. Future research should analyze velar morphology in participants with repaired cleft palate and/or OSA, as well as implementing a longitudinal study design.

\section{References}

1. Deepa V, David CM, Ramnarayan BK. Morphological varieties of soft palate in normal individuals, cleft palate patients and obstructive sleep apnea patients with reference to Indian population: a preliminary digital cephalometric study. World J Dent 2013; 4: 241-9.

2. Deshmukh RA, Bagewadi AS. Morphometric evaluation and comparison of soft palate in individuals with and without oral submucous fibrosis: a digital cephalometric study. SRM J Res Dent Sci 2015; 6: 220-4.

3. Raja Lakshmi C, Ayesha Thabusum D, Bhavana SM. An innovative approach to evaluate the morphological patterns of soft palate in oral submucous fibrosis patients: a digital cephalometric study. Int J Chronic Dis 2016; 2016: 5428581.

4. Mohan RS, Verma S, Singh U, Agarwal N. Morphometric evaluation of soft palate in oral submucous fibrosis - a digital cephalometric analysis. West Afr J Radiol 2014; 21: 7-11

5. Pépin JL, Veale D, Ferretti GR, Mayer P, Lévy PA. Obstructive 
sleep apnea syndrome: hooked appearance of the soft palate in awake patients - cephalometric and CT findings. Radiology 1999; 210: 163-70.

6. You M, Li X, Wang H, Zhang J, Wu H, Liu Y, et al. Morphological variety of the soft palate in normal individuals: a digital cephalometric study. Dentomaxillofac Radiol 2008; 37: 344-9.

7. Guttal KS, Breh R, Bhat R, Burde KN, Naikmasur VG. Diverse morphologies of soft palate in normal individuals: a cephalometric perspective. J Indian Acad Oral Med Radiol 2012; 24 : 15-9.

8. Kumar DK, Gopal DK. Morphological variants of soft palate in normal individuals: a digital cephalometric study. J Clin Diagn Res 2011; 5: 1310-3.

9. Nagaraj T, Goswami RD, James L, Sreelakshmi N, Veerabasavaiah BT, Shruthi R. A radiographic assessment of morphologies of soft palate: a retrospective study. J Med Radiol Pathol Surg 2016; 3: 1-4.

10. Niu YM, Wang H, Zheng Q, He X, Zhang J, Li XM, et al. Morphology of the soft palate in normal humans with digital cephalometry. Hua Xi Kou Qiang Yi Xue Za Zhi 2006; 24: 321-7.

11. Praveen BN, Amrutesh S, Pal S, Shubhasini AR, Vaseemuddin S. Various shapes of soft palate: a lateral cephalometric study. World J Dent 2011; 2: 207-10.

12. Santosh VK, Singh P, Pagare SS. Normative soft palate dimensions and morphology in a subset of Indian population: a digital cephalometric study. Indian J Oral Health Res 2015; 1: 48-51.

13. Shankar VN, Hegde K, Ashwini NS, Praveena V, Ravi Prakash SM. Morphometric evaluation of soft palate in oral submucous fibrosis - a digital cephalometric study. J Craniomaxillofac Surg 2014; 42: 48-52.

14. Verma P, Verma KG, Kumaraswam KL, Basavaraju S, Sachdeva SK, Juneja S. Correlation of morphological variants of the soft palate and Need's ratio in normal individuals: a digital cephalometric study. Imaging Sci Dent 2014; 44: 193-8.

15. Khaitan T, Pachigolla R, Uday G, Balmuri PK, Chennoju SK, Pattipati S. Digital cephalometric analysis illustrating morphological variation of the soft palate. J Indian Acad Oral Med Radiol 2015; 27: 532-8.

16. Agrawal P, Gupta A, Phulambrikar T, Singh SK, Sharma BK, Rodricks D. A focus on variation in morphology of soft palate using cone-beam computed tomography with assessment of
Need's ratio in central Madhya Pradesh population. J Clin Diagn Res 2016; 10: ZC68-71.

17. More CB, Saha N, Vijayvargiya R. Morphological study of soft palate by using computed tomography - a prospective study. $\mathbf{J}$ Clin Diagn Res 2015; 9: ZC71-4.

18. Perry JL, Kuehn DP, Sutton BP, Gamage JK, Fang X. Anthropometric analysis of the velopharynx and related craniometric dimensions in three adult populations using MRI. Cleft Palate Craniofac J 2016; 53: e1-13.

19. U.S. Food and Drug Administration [Internet]. Silver Spring, MD: The Administration; [cited 2019 Jan 31]. Benefits and risks. Available from: https://www.fda.gov/Radiation-EmittingProducts/RadiationEmittingProductsandProcedures/MedicalImaging/MRI/ucm482765.htm.

20. Bae Y, Kuehn DP, Sutton BP, Conway CA, Perry JL. Three-dimensional magnetic resonance imaging of velopharyngeal structures. J Speech Lang Hear Res 2011; 54: 1538-45.

21. Perry JL, Sutton BP, Kuehn DP, Gamage JK. Using MRI for assessing velopharyngeal structures and function. Cleft Palate Craniofac J 2014; 51: 476-85.

22. Mason KN, Perry JL, Riski JE, Fang X. Age-related changes between the level of velopharyngeal closure and the cervical spine. J Craniofac Surg 2016; 27: 498-503.

23. Perry JL, Kollara L, Kuehn DP, Sutton BP, Fang X. Examining age, sex, and race characteristics of velopharyngeal structures in 4- to 9-year-old children using magnetic resonance imaging. Cleft Palate Craniofac J 2018; 55: 21-34.

24. Senaratna CV, Perret JL, Lodge CJ, Lowe AJ, Campbell BE, Matheson MC, et al. Prevalence of obstructive sleep apnea in the general population: a systematic review. Sleep Med Rev 2017; 34: 70-81.

25. Bixler EO, Vgontzas AN, Lin HM, Liao D, Calhoun S, Vela-Bueno A, et al. Sleep disordered breathing in children in a general population sample: prevalence and risk factors. Sleep 2009; 32: 731-6.

26. Perry JL. Variations in velopharyngeal structures between upright and supine positions using upright magnetic resonance imaging. Cleft Palate Craniofac J 2011; 48: 123-33.

27. Kollara L, Perry JL. Effects of gravity on the velopharyngeal structures in children using upright magnetic resonance imaging. Cleft Palate Craniofac J 2014; 51: 669-76. 